\title{
La construcción de la identidad política en las elecciones presidenciales ecuatorianas 2021
}

\section{Caso Yaku Pérez, spot publicitario "Viene Lo Nuevo. Viene Lo Bueno"}

\section{The construction of political identity in the 2021 Ecuadorian presidential elections}

\author{
Yaku Pérez case, advertising spot "Viene Lo Nuevo. Viene Lo Bueno"
}

(New things are coming, Good is coming)

DOI: https://doi.org/10.29166/tyc.v1i22.2968

\section{Juan Esteban Baldeón Salazar}

Estudiante de Comunicación Social en la Universidad Central del Ecuador. Se ha desempeñado como periodista en medios digitales y asistente de investigación en el Centro de Estudios e Investigaciones Especializadas (CIESS). Sus preferencias de estudio son la comunicación política y las redes sociales.

Correo: jebaldeon@uce.edu.ec

\section{Samantha Romero Castañeda}

Socióloga con mención en Desarrollo de la Pontificia Universidad Católica del Ecuador (PUCE). Tiene una especialización en Comunicación Política Digital.

Correo: sammyromer01905@gmail.com

\section{Resumen}

El presente texto analiza la construcción de identidad política realizada por el candidato presidencial Yaku Pérez, previo a las elecciones presidenciales de Ecuador de 2021. Para ello, se utiliza el spot publicitario titulado "Viene Lo Nuevo, Viene Lo Bueno", lanzado en diciembre del año 2020, como objeto de análisis. El estudio hace posible la identificación de elementos simbólicos y discursos que evidencian una construcción coherente de la identidad política del candidato.

Palabras clave: Yaku Pérez, identidad política, comunicación política, análisis del discurso.

\section{Abstract}

The present text analyzes the creation of a political identity, used by presidential candidate Yaku Pérez prior to the presidential Ecuadorian elections in 2021. For that matter, the advertising spot called "Viene Lo Nuevo, Viene Lo Bueno", launched in December 2020, has been used as an object of analysis. This study allows us to identify the symbolic elements and discourses that shed light over a coherent creation of the candidate's political identity.

Keywords: Yaku Pérez, political identity, political communication, discourse analysis. 


\section{Introducción}

A partir de los últimos meses del 2019 en Ecuador arrancó el proceso preelectoral. En diciembre, la Asamblea Nacional tramitó las reformas al Código de la Democracia, aplicadas durante los comicios del 2021, en los cuales se elegiría al futuro binomio presidencial, a los próximos asambleístas y a los representantes al Parlamento Andino.

El 12 de marzo del 2020, el Consejo Nacional Electoral (CNE) - puesto en funciones a través de un Consejo de Participación Ciudadana Transitorio - proclamó el inicio del periodo electoral. Para septiembre y octubre de ese año, el CNE tramitó la inscripción de candidaturas de los aspirantes a dignidades públicas y del 31 de diciembre del 2020 al 04 de febrero del 2021, el CNE abrió el proceso de campaña política.

El o7 de febrero del 2021, los ecuatorianos acudieron a las urnas y manifestaron su voto. Allí, se vieron representadas las principales fuerzas políticas. El candidato por el Movimiento Unión por la Esperanza (UNES), auspiciado por el correísmo y autodefinido como progresista, Andrés Arauz, alcanzó el liderazgo de esta primera vuelta con el $32.72 \%$ de votos. El segundo lugar estuvo en disputa entre el candidato Guillermo Lasso, candidato de la banca y representante del partido CREO, y Yaku Pérez, el representante del movimiento indígena auspiciado por el movimiento político $\mathrm{Pa}$ chakutik. Tras ambientes de pugnas y tensiones en las instituciones del Estado, el CNE proclamó ganador a Lasso con un 19.74\% de votos. El tercer lugar fue ocupado por el candidato Pérez, con un 19.39\%.
A continuación, el presente estudio propone analizar la identidad política que Yaku Pérez construyó a través de su spot electoral "Viene Lo Nuevo. Viene Lo Bueno".

\section{Comunicación política}

La comunicación política, como práctica, es tan antigua como la revolución cognitiva. Su génesis puede encontrarse desde que el ser humano ejerció poder sobre sus semejantes. El poder, en tanto relación y no atributo, siempre requiere de procesos de significación o violencia que legitimen su ejercicio. La comunicación aparece como la base de estas relaciones y, por ende, como elemento de cohesión de todo grupo social. Desde la Antigua Grecia hasta la modernidad, la comunicación y la política son dos ámbitos imbricados en el ejercicio del poder. Después de todo, "la comunicación adquiere un papel trascendental, cuyas funciones son informar, interpretar y mediar intereses" (Reyes, O'Quinn, \& Morales, 2011, p. 84).

A partir de 1970, la comunicación política atrae el interés de académicos y gobernantes para consolidarse como una disciplina, cuyo hito más importante sería la creación de la división de estudios de comunicación política por parte de la Asociación Internacional de Comunicación, en 1973. A partir de entonces, el impulso de sociedades de mercado y los procesos de racionalización de la administración pública desembocaron en la diferenciación de dos ámbitos de la joven disciplina: la comunicación política electoral y la comunicación gubernamental. Ambos ámbitos se diferencian por su función rectora. El objetivo de la comunicación guberna- 
mental es promover el consenso entre la ciudadanía para legitimar, a través de procesos de producción y difusión de bienes simbólicos, las instancias gubernamentales del poder. Por su parte, la comunicación política electoral, a través de elementos similares a su ámbito vecino, pretende seducir al mayor número posible de electores durante un periodo de campaña. Al ser pertinente para este estudio, se ofrece un breve abordaje conceptual de la comunicación política electoral.

A finales del siglo XX la comunicación electoral recibe el impulso del emergente proyecto neoliberal, entendido como "una forma particular de razón que configura todos los aspectos de la existencia en términos económicos" (Brown, 2015, p. 13). Esta nueva racionalidad, orientada a la economización de todas las esferas de la vida humana, impulsa la idea de que el elector es equivalente a un consumidor y la ciudadanía a un mercado. La política, por tanto, debe migrar del sólido espacio de las convicciones y la militancia, a un espacio de flujos basado en las ofertas, el entretenimiento y el gusto. En ese sentido, "la comunicación política en tiempos de la mediatización no podría ser sino una estrategia de la mentira" (Cuadra, 2009, pág. 28). De allí que la comunicación política electoral tome por punta de lanza al denominado marketing electoral y aplique sus principios a la construcción de un candidato como un producto más dentro de una sociedad de mercado.

\section{Identidad política}

Desde la perspectiva comunicacional, toda identidad es un proyecto narrativo basado en el lenguaje. Al ser un proyecto, no se puede asumir que su por- tador la tenga por esencia inamovible de su "yo". Al contrario, un proyecto identitario es un proceso, no un resultado. Se articula en forma de narrativa, pues constituye una relación entre el "yo" y las estructuras discursivas de su entorno. Es un dar sentido y hacer accesible a la experiencia las relaciones entre el mundo objetivo e intersubjetivo que rodean al portador de esta narrativa identitaria. $\mathrm{Su}$ base es el lenguaje, institución social totalitaria que permite la existencia del individuo como sujeto "sujetado", es decir, "atado a las relaciones de poder, de significación y de producción del saber” (Velasco, 2014, p. 7).

Este enfoque de la identidad ha sido atendido por diversos autores, haciendo énfasis en el lenguaje como sustento de narrativas identitarias.

Desde la tradición hermenéutica, John Thompson (2010) propone su visión del "yo" y, consecuentemente, de la identidad como un proyecto simbólico. Este autor la define como un proyecto que el individuo construye activamente, a partir del repertorio de materiales simbólicos que se le ofrecen en su entorno y, con esta profusión de ofertas identitarias, "el individuo teje una explicación coherente de quién es él o ella, una narrativa de la propia identidad" (p. 273). Por supuesto, el acceso de los sujetos al distinto material simbólico es asimétrico y depende de procesos históricos y de las condiciones materiales de vida de cada uno de ellos. De igual forma, las ofertas identitarias están atadas tanto a estructuras económicas y a superestructuras políticas como a sucesivas coyunturas, que, en última instancia, van a condicionar las bases de un posible proyecto identitario.

En otros estudios, la identidad aparece más directamente vinculada a su 
condición de narrativa. Desde un enfoque cercano al psicoanálisis y orientado al estudio del "yo" en el mundo virtual, el sujeto es conceptualizado como "una unidad ilusoria construida en el lenguaje, a partir del flujo caótico y múltiple de cada experiencia individual" (Sibilia, 2017, p. 37). De tal forma que una narrativa identitaria es una forma de ordenar y habitar este "flujo caótico". De ello, se desprende que el "yo" es "una ficción gramatical, un centro de gravedad narrativa, un eje móvil e inestable donde convergen todos los relatos de uno mismo" (Ibid.). Por tanto, esta perspectiva, termina por definir al yo como "cruce de narrativas" (Ibid., p. 38). $Y$ mientras estas adquieran cierto nivel de concreción para ser formas de estar en el mundo, se podría hablar de narrativas identitarias. De igual forma, la perspectiva comunicacional, cercana al ámbito pragmático, sugiere que estas solo existen en tanto son expuestas y reconocidas por un grupo social. Como exponen desde los teóricos de Palo Alto hasta García Márquez, aquello que no se comunica no existe. Por tanto, "toda identidad necesita mostrarse, comunicarse para hacerse real, lo cual implica, por parte del actor individual o colectivo, la utilización dramatúrgica de aquellas marcas, atributos y elementos que le permitan desplegar su identidad" (Reguillo, 2013, p. 78). Una narrativa identitaria adquiere plena vigencia solo en tanto se comunica, a través de puestas en escena y, lógicamente, de discursos.

Fundamentalmente, una narrativa identitaria se pone en escena a través de una acción dramatúrgica, en tanto "encuentro en el que los participantes constituyen los unos para los otros un público visible y se representan mutuamente algo" (Habermas, 2014, p. 124).
Desde este bagaje teórico, se puede dimensionar la posibilidad de una identidad política. Para el presente trabajo, se concibe como una narrativa abastecida con amplio material simbólico. Tiene anclaje en procesos históricos, partidos políticos 0 , en los entornos más mediatizados, en flujos de contenido con los que un político se representa a sí mismo como un personaje dentro su propia narrativa. Pese a estos anclajes, con temporalidad propia, este tipo de identidad se promociona fuertemente en periodos electorales y su objetivo es generar la mejor imagen posible. Después de todo, en comunicación política electoral, toda identidad política genera una imagen del candidato, en tanto construcción perceptiva de las audiencias.

Por ello, valdría señalar que la identidad política no solo se construye sobre acciones dramatúrgicas, sino, a la vez, sobre estrategias orientadas a la consecución de los objetivos de comunicación política, gubernamental o electoral. Es decir, toda identidad política también se nutre, abiertamente, del ejercicio de una acción instrumental.

\section{Marco metodológico}

Para el presente estudio, se ha seleccionado el spot publicitario "Viene Lo Nuevo, Viene Lo Bueno", que corresponde a la campaña electoral presidencial del 2021 del candidato Yaku Pérez. El spot tiene una duración de 54 segundos, ideal para la difusión en redes sociales, y fue presentado el 2 de diciembre de 2020, junto con el hashtag \#VieneLoNuevoVieneLoBueno. El spot servirá como objeto de estudio para el análisis de las narrativas identitarias digitales en el marco de la 
política ecuatoriana. Los criterios que guiaron la selección de esta pieza audiovisual fueron la alta difusión que tuvo entre los electores y la profundidad con la que se manejó la construcción identitaria del candidato Pérez en este breve spot.

Para el análisis, se ha planteado el uso de una metodología cualitativa, a través de la técnica de análisis del discurso. Esta técnica ha sido escogida, dado que, "junto con sus funciones descriptivo/representacionales, el lenguaje adquiriría, por lo tanto, un carácter productivo, presentándose como un elemento formativo de realidades." (Ibáñez, 2011, p. 22). De este modo, el análisis del discurso ofrece la posibilidad de analizar el spot publicitario desde perspectivas estilísticas, retóricas e ideológicas.

El enfoque cualitativo y las herramientas ofrecidas por el análisis del discurso permiten, además, situar al spot publicitario no como un producto comunicacional aislado, sino como un producto situado en contexto y orientado a metas. La identificación del contexto en el que fue creado y distribuido hace posible una lectura de la realidad social y del discurso en su capacidad generativa.

El análisis del discurso aplicado al presente estudio requirió la identificación de un nivel actancial, un nivel narrativo y un nivel discursivo. Es así como se plantea la ubicación y el análisis de personajes, relatos y elementos identitarios en el spot publicitario "Viene Lo Nuevo, Viene Lo Bueno".

\section{Unidad de análisis: el spot "Viene lo Nuevo Viene Lo Bueno" (2021)}

El spot Viene lo Nuevo Viene lo Bueno inicia con un plano medio de una persona vestida con camisa, sosteniendo máscaras sobre su cara. El plano va acompañado de música instrumental suave y una narración en off del candidato Yaku Pérez. Durante el primer segmento, la voz en off narra lo siguiente, "Correa lo puso a Moreno. Moreno lo puso a Lasso, que co-gobernó con él. Y ahora, Lasso dice que él es el cambio y Correa dice que Arauz es la esperanza, como lo era Lenin" (Pérez, 2020). Durante la narración, la persona va cambiando máscaras que sostiene sobre su cara, las cuales representan a los personajes de la política ecuatoriana mencionados, en el siguiente orden: el expresidente Rafael Correa, el presidente Lenin Moreno (en el momento de la campaña), el candidato a la presidencia por CREO, Guillermo Lasso, a Rafael Correa, por segunda vez, y al candidato a la presidencia por UNES, Andrés Arauz.

En esta primera sección del spot, existe una evidente intención de ataque hacia los principales oponentes del candidato en los comicios del 2021: Guillermo Lasso y Andrés Arauz. Para ello, el equipo de Pérez opta por representar una suerte de complicidad entre sus oponentes actuales y los presidentes de los dos últimos gobiernos del Ecuador. Establece una narrativa que los sitúa como culpables de los problemas del país y, también, los relega al pasado de la política ecuatoriana. En el caso del candidato Andrés Arauz, la estrategia es ligeramente distinta, pues se trata de una imagen relativamente nueva en el escenario político nacional. Es así que el equipo de Pérez opta por apelar a una mala experiencia del pasado. De este modo, le recuerda al espectador que, en las elecciones del año 2017, la candidatura de Lenin Moreno fue respaldada por el expresidente Correa, sin resultados óptimos en su gestión. En el spot, se pre- 
tende identificar a la candidatura de Arauz con el escenario político de fragmentación de los últimos años. De esta forma, prepara al espectador para un mensaje de cambio en la segunda sección.

A los 14 segundos de iniciado el material, da comienzo el segundo segmento del mismo. En él, se muestra al candidato a la presidencia por Pachakutik, sentado frente a un fondo verde que resembla árboles y plantas de un escenario natural. El candidato en cuestión viste una camisa sencilla de color celeste. Alrededor de su cuello, lleva un pañuelo con el patrón de la wiphala, misma que lleva los colores rojo, naranja, amarillo, verde, celeste, azul y morado. Esta se considera un símbolo de los pueblos andinos y ha sido tomada también como símbolo del movimiento político al que representa Pérez. Sobre el pañuelo, además, luce un collar con la figura de la chakana o cruz andina, la cual simboliza el pensamiento y la cosmovisión de los pueblos andinos. En este segmento del spot publicitario, el candidato habla directamente a la cámara y señala,

Yo sé que estás cansado de caretas y de ver siempre a los mismos y de que nos mientan. Yo también. Vengo a invitarte a soñar un país mejor, el que nos merecemos. Y a hacerlo juntos, cuidando nuestro planeta y lo más valioso que tenemos: eres tú y cada uno de los ecuatorianos. Un país con trabajo, salud, educación y oportunidad para todos. Te invito a que enterremos el pasado, cambiemos para siempre nuestra historia (Pérez, 2020).

En este espacio, el candidato se presenta abiertamente. Palabra e imagen acompañan la puesta en escena de su persona y la presentación de su propuesta. Pérez apela a la idea de lo auténtico, diferenciándose de esos políticos que su spot asocia con la falsedad de una máscara. Después, el candidato empatiza con los ciudadanos-consumidores, les atribuye un cansancio compartido de ese tipo tradicional de políticos. Finalmente, les ofrece su propuesta: un país diferente, que se distingue de ese pasado monótono, lineal y unidireccional. Seduce con la idea de una verdadera ruptura que cambie la historia del Ecuador hacia el desarrollo. Si en el primer segmento Pérez construye a sus enemigos, aquí edifica su identidad en relación con los otros y enfatiza en su condición distintiva.

La última sección del spot tiene una duración de 7 segundos. En ella, se muestra un fondo de color azul marino. Sobre él, en el centro de la pantalla, se sitúan las palabras, "Yaku Presidente" y "Vota 18" con una tipografía sencilla en color blanco, acompañadas solo por una franja segmentada con los colores rojo, naranja, amarillo, verde, celeste, azul y morado. En la esquina inferior izquierda, aparece progresivamente la etiqueta \#VieneLoNuevoVieneLoBueno. En la esquina inferior derecha, aparece, por única vez en todo el spot, el logotipo del movimiento Pachakutik. Esta vez, una voz en off distinta cierra el diciendo: "Yaku. Viene lo nuevo, viene lo bueno."

A casi 3 meses de su lanzamiento, el spot cuenta con 232 mil 652 reproducciones en la plataforma YouTube, $271 \mathrm{mil}$ 780 reproducciones en la plataforma Instagram, 32,6 mil reproducciones en la plataforma Twitter, y 1.8 millones de reproducciones en la plataforma Facebook. En todas las plataformas, el producto audiovisual fue acompañado por una corta descripción que reza "iBasta de caretas! Viene el país que soñamos y que nos merecemos. Viene un país mejor." La descripción también fue acompañada, en 
todas las plataformas, por un emoticono de la bandera ecuatoriana y un emoticono del arcoíris. Así también, el mensaje fue acompañado por los hashtags \#VieneLoNuevoVieneLoBueno, \#YakuEsPueblo, \#Pachakutik y \#Lista18 en las plataformas de YouTube, Facebook e Instagram. En Twitter, el video fue acompañado solo por los 2 primeros hashtags.

\section{La construcción del rival político}

De entrada, el spot empieza con un discurso en forma de narrativa. Se cuenta al espectador un resumen de los últimos 15 años a través de la sucesión de las personas en el poder: "Correa lo puso a Moreno. Moreno lo puso a Lasso, que cogobernó con él”. Así, el video presenta una historia que simplifica profundos procesos históricos en una sucesión de personajes que fueron puestos en el poder. Se ataca a Correa, culpándolo por el gobierno de Moreno y a Lasso, evocando sus relaciones con el presidente Moreno. La reiteración del verbo "puso", en tercera persona del pretérito del modo indicativo, implica a estos tres personajes dentro una misma línea narrativa. En suma, todos aparecen vinculados a un mismo pasado, monótono, lineal y negativo. El storytelling y la simplificación aparecen como recursos retóricos y preparan el terreno para la posterior presentación del candidato.

Adicionalmente, esta historia se cuenta a través de un elemento con alta carga simbólica: la máscara. Después de ver a Correa, Moreno y Lasso, aparece la máscara de Arauz y, por segunda vez, la de Correa. El spot proyecta la sombra del pasado sobre el candidato de UNES. Sin embargo, todos ellos aparecen represen- tados en máscaras. Una máscara oculta, disfraza y permite excesos. Es un elemento que anula la identidad de su portador. Adicionalmente, este signo aparece vinculado al engaño, el anonimato y el crimen. Además, en usos rituales, la máscara suele representar personajes que inspiran temor. En el spot, se aplican estos simbolismos y se mutila la autenticidad de todos los políticos representados. Se construye su identidad negativamente, se los vincula a un mismo modo de hacer política y se los proyecta como amenaza.

De esta forma, el video plantea un problema, cuyo punto de inflexión será la abierta presentación del candidato Yaku Pérez, quien edifica su mensaje en relación con estos antecedentes y los toma como punto de partida para su construcción identitaria.

\section{Yaku: de político a ciudadano}

El anuncio publicitario \#VieneLoNuevoVieneLoBueno presenta un punto de quiebre en su segunda sección. A diferencia del primer segmento, enfocado en una estrategia para posicionar una identidad de contraste, este segmento se orienta a construir la imagen identitaria de Yaku Pérez, a través del uso de herramientas simbólicas en su discurso. En términos generales, pretende construir una imagen del candidato relacionado con una nueva, y mejor forma de hacer política, apelando directamente al elector.

El escenario utilizado para este segundo momento del spot merece ser uno de los elementos de análisis. La transición desde la primera sección pasa de un fondo blanco, artificial y propio de un set, a un ambiente que retrata la natura- 
leza y en el cual predomina el color verde de los árboles. En términos generales, se puede decir que esta elección corresponde a la postura ecologista que el candidato quiere posicionar como parte de su identidad política. Sin embargo, el cambio entre el primer y el segundo escenario también evoca un signo de autenticidad que posiciona en su discurso. Como ya se ha mencionado, a lo largo del spot existe una intención de deslegitimar la autenticidad de quienes representan la oposición a Pérez. Cuando se los menciona y se los retrata, todos se encuentran ubicados en un escenario frío y artificial. Sin embargo, el representante de Pachakutik aparece en un escenario que resembla la naturaleza, sentado en una postura relajada, y hablando directamente hacia la cámara. Todas estas disposiciones performativas comunican las cualidades de autenticidad y cercanía que el candidato pretende potenciar como parte de su identidad política.

Esta intención por construir una narrativa de autenticidad se presenta, también, en las palabras cuidadosamente elegidas y pronunciadas por Pérez, quien inicia su intervención diciendo: "Yo sé que estás cansado de caretas y de ver siempre a los mismos y de que nos mientan. Yo también.” Al hacerlo, no sólo deslegitima una vez más a sus oponentes, sino que se ubica a sí mismo desde una perspectiva de ciudadano, no de político, que evidencia y se lamenta por la forma en que ha sido manejada la política ecuatoriana de los últimos años. La lexía "Yo también" identifica a Yaku Pérez, a través del pronombre personal, con millones de ciudadanos que están cansados de formas políticas tradicionales, con quienes la identidad del candidato colinda a través del adverbio aditivo "también".
Después, el candidato extiende una invitación al elector a trabajar juntos en beneficio del país. Así, rompe con la imagen tradicional del político mesiánico, capaz de solventar todos los problemas de los electores, y se ubica, una vez más, al nivel de los demás ciudadanos. En este punto, incluso se muestra abierto y receptivo a la ayuda para su Gobierno. Esto, sin embargo, no debilita su identidad política, sino que pretende construir una narrativa de cercanía con la ciudadanía, algo muy valorado en el contexto de las elecciones del 2021, enmarcadas en una fuerte desconfianza sobre las instituciones y los servidores del Estado.

A través de esta corta pero clara intervención, Pérez rompe con los patrones típicos de la forma de hacer política. Incluso, se atreve a mencionar brevemente cuáles son las prioridades en su plan de gobierno: la naturaleza y los ecuatorianos. Esta elección de sustantivos comunes y de amplio alcance apela directamente al espectador, a quien se pretende identificar como protagonista. Nuevamente, a través de su discurso, el candidato opta por no potenciar su identidad como político, sino como ciudadano ecuatoriano. Algo que, si bien no corresponde al presente estudio, ha realizado también a través de otras estrategias electorales, tal como el haber denominado a su programa de gobierno "Minka por la Vida”. En kichwa, el vocablo "Minka" significa trabajo colaborativo.

A lo largo de esta sección del spot merece reconocerse, también, el uso adecuado de objetos de carga simbólica para transmitir mensajes más allá del mensaje oral. Estos objetos son parte de la puesta en escena de la identidad de Pérez y, como todo lo demás, comunican. El uso de accesorios con símbolos relacionados al mundo andino y la cosmovisión indí- 
gena, tales como la whipala y la chakana, sumados al uso ya mencionado de un escenario representativo de la naturaleza, mantienen presente a lo largo del spot el compromiso del candidato no solamente con ofertas electorales, sino con su propia postura política e ideológica. Este uso de símbolos no pretende generar en el espectador solamente una relación directa con el movimiento político al que Pérez representa, sino también, y principalmente, una relación de apego hacia aquello que simboliza las raíces y los orígenes de la sociedad ecuatoriana, con especial énfasis en el sector andino.

En suma, las elecciones simbólicodiscursivas tomadas por Pérez y su equipo de comunicación presentan la construcción de una identidad política coherente, afincada en la cosmovisión andina, pero renovada como el rostro auténtico del cambio en el escenario político. Una identidad que, además, utiliza al propio espectador/elector como punto de equiparación del candidato, pues trata de asemejar sus posturas, deseos e intenciones a aquellos generalizados en el imaginario social de la sociedad ecuatoriana.

\section{Una identidad seductora}

En un tercer momento, el spot presenta su desenlace. Allí, aparecen elementos lingüísticos de relevancia, pues se expone el texto "\#VieneLoNuevo.VieneLoBueno", mientras, de modo simultáneo, una voz en off le da lectura. A través de esta micro unidad de análisis, se puede evidenciar la narrativa identitaria de Pérez, el texto en cuestión evoca una construcción inclusive estética de la propuesta. Se construye una identidad seductora, como es propio de la moderna forma de comunicación política. Hay una intencionalidad estratégica tanto en la forma del mensaje, como en su contenido. Por ejemplo, a nivel de forma, hay un evidente uso de recursos retóricos, como la anáfora, consagrada en la repetición de la frase "Viene Lo", el símil entre lo "Nuevo" y lo "Bueno" y, finalmente, la rima entre ambas palabras.

Estas estrategias de superficie permiten presentar la identidad de Pérez del modo más narrativamente atractivo posible. Además, el uso del verbo venir en la tercera persona del presente indicativo evoca esperanza. Se comunica la certeza, por ello ni siquiera se usa una conjugación en tiempo futuro, de que algo, en este caso un proyecto político, viene. Finalmente, si la identidad política de Yaku está anclada y edificada sobre este proyecto político que "viene", es lógico suponer que "Bueno" y "Nuevo" son adjetivos calificativos asociados a la identidad del propio candidato. Se apela al imaginario común de asociar, inmediatamente, lo bueno con lo nuevo. En la propuesta de Pérez, lo "Nuevo" seduce, apelando directamente a un mercado político que puede valorar su identidad como una mercancía novedosa. Por su parte, lo "Bueno" justifica y complementa la oferta inicial.

Finalmente, el spot muestra, por única vez, el logo de Pachakutik e invita a votar por la lista 18. La identidad política de Pérez, por tanto, se ve respaldada en estructuras orgánicas, vinculadas a una política de convicción y basada en partidos. Sin embargo, estos elementos no son centrales. Tienen un espacio mínimo. Lo primordial, en la oferta identitaria de Pérez, no es el sólido anclaje de su partido, sino, más bien, cualidades más seductoras, como la novedad y la calidad de su propia identidad política. 


\section{\#YakuEsPueblo}

Por último, resulta de gran importancia notar cómo algunos de los hashtags utilizados en el proceso de difusión del spot publicitario apuntan a consolidar los mensajes identitarios transmitidos a lo largo del mismo. Si bien ya se han analizado las implicaciones del hashtag \#VieneLoNuevoVieneLoBueno, cabe también prestar atención a otra etiqueta que acompañó al producto audiovisual en todas las plataformas de distribución: \#YakuEsPueblo.

Con este hashtag se pretende, una vez más, fortalecer una identidad de cercanía a la ciudadanía ecuatoriana. En este caso, la construcción lingüística es, en forma, una metáfora. Sin conectores de por medio, la asociación de ambos términos se presupone como totalmente equivalente. Así, se asimila al conjunto de electores bajo la palabra "pueblo", vocablo que está vinculado no solo a las grandes mayorías sino, a su vez, a un sujeto histórico colectivo. Así, Pérez implica en su discurso a un nuevo protagonista de un posible gobierno, formado por quienes han sido excluidos de participar en la toma de decisiones pero que, al mismo tiempo, son los más afectados por las cosas que suceden en el escenario político.

La etiqueta, además, usa solamente el primer nombre del candidato, evitando posicionarlo como un personaje distante y formal, tal como sería el trato entre el elector y un familiar o un amigo cercano. En suma, pretende comunicar, en solo 3 palabras, la identidad política que se ha construido del candidato a lo largo del anuncio y de su campaña electoral.

\section{Conclusiones}

Durante las elecciones del 2021, se evidenció el uso de estrategias de comunicación política para posicionar a los distintos candidatos. Estas formas de comunicación instrumental se orientaron a la construcción de una identidad política, basada en discursos, tanto simbólicos como propiamente lingüísticos, difundida a través de spots tradicionales y redes sociales. El caso de Yaku Pérez ofrece una perspectiva respecto a la importancia de ejecutar estrategias de comunicación políticas coherentes con la representación identitaria del candidato, construida a través de narrativas. De tal forma, este estudio puede atribuir al candidato Pérez un manejo de su identidad política de cara a los comicios 2021.

Entre los méritos consolidados en la narrativa identitaria de Pérez en el spot sujeto a análisis, se puede destacar tanto el uso de estrategias de fondo (storytelling), como estrategias de forma (simplificación, metáforas y recursos estilísticos). Esta diversidad de elementos encontró una articulación coherente y, a la vez, seductora en la narrativa del spot "Viene Lo Nuevo. Viene Lo Bueno".

El equipo de comunicación política de Pérez construyó la representación de un candidato cercano a la ciudadanía y que empatiza con sus necesidades y problemas. De esta forma, rompió con la imagen tradicional y paternalista de la política ecuatoriana, poniendo al candidato al mismo nivel del elector.

Además de una construcción identitaria basada en las características propias del candidato, el equipo de Pérez logró crear una narrativa identitaria en torno al contraste y el rechazo a otras fi- 
guras de la política ecuatoriana. Es decir, se apuntaló una forma de identidad relacional, cuyo valor estribó en una posición estratégica respecto a sus propios rivales. De este modo, construyeron también una narrativa de oposición capaz de marcar distancias con las corrientes y partidos tradicionales del Ecuador, presentando al candidato Pérez como una tercera vía dentro del proceso electoral. 


\section{Bibliografía}

Brown, W. (2015). El pueblo sin atributos: La revolución secreta del neoliberalismo. Ciudad de México: Malpaso.

Cuadra, Á. (2009). La comunicación política en la era digital. Centro Gumilla Comunicación, 22-32.

Habermas, J. (2014). Teoría de la Acción Comunicativa. Madrid: Trotta.

Ibáñez, T. (2011). El giro linguístico. En L. Î̃iguez, Análisis del discurso. Manual para las ciencias sociales (págs. 9-25). Madrid: Editorial UOC.

Pérez, Y. (2 de Diciembre de 2020). Viene Lo Nuevo, Viene Lo Bueno. Spot campaña electoral 2021, Ecuador. https://www.facebook.com/yakuperezoficial/videos/vienelonuevovienelobueno/382838319653107/

Reguillo, R. (2013). Culturas juveniles. Estrategias políticas del desencanto. Ciudad de México: Siglo XXI.

Reyes, M. C., O'Quinn, J. A., \& Morales, J. M. (2011). Reflexiones sobre la comunicación política. Espacios Públicos, vol. 14 , num. 30, 85-101.

Sibilia, P. (2017). La intimidad como espectáculo. Ciudad de México: Fondo de Cultura Económica.

Thompson, J. (2010). Los media y la modernidad. Ciudad de México: Paidós Comunicación.

Velasco, M. G. (2014). Lenguaje, sujeto y subjetividad: Tras los hilos de las palabras. Revista de Ciencias de la Educación Academicus, 4-9. 